eventually became long-term donors. 32 donors provided 2009 times of fecal donations. As shown in Figure 3 (IDDF2021-ABS-0086 Figure 3. Correlation of fecal weight and amount of enriched washed microbiota), the fecal weight was not well correlated with the amount of enriched washed microbiota (95\% CI, 0.62-0.67, P $<0.0001$ ), r was 0.64 , (IDDF2021-ABS-0086 Figure 4. Detailed exclusion criteria and proportions).

Conclusions In our center, we selected 32 donors from 944 students, with a qualified rate of $3.4 \%$. This result is similar to the qualified rate of donor screening reported in stool

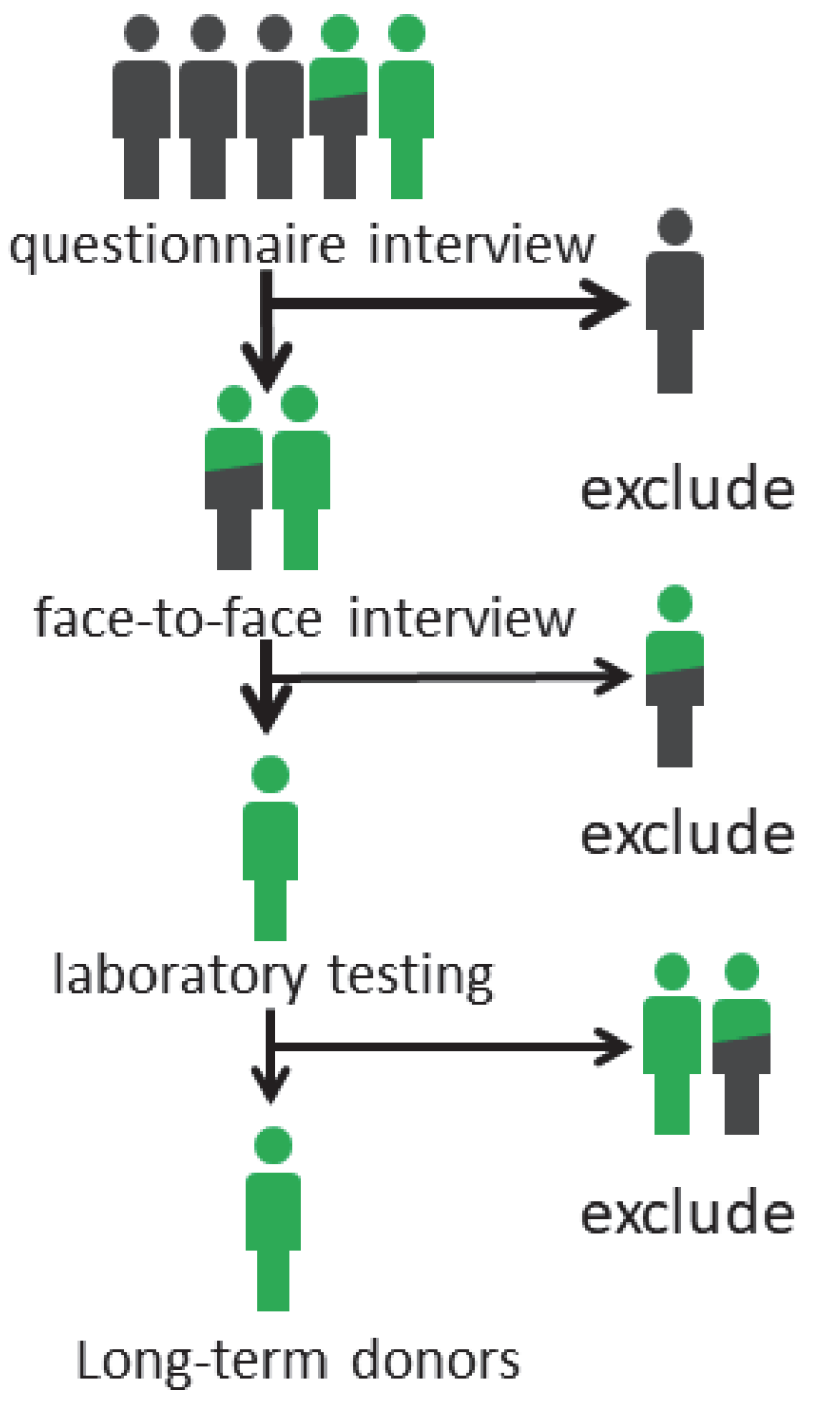

Abstract IDDF2021-ABS-0086 Figure 1

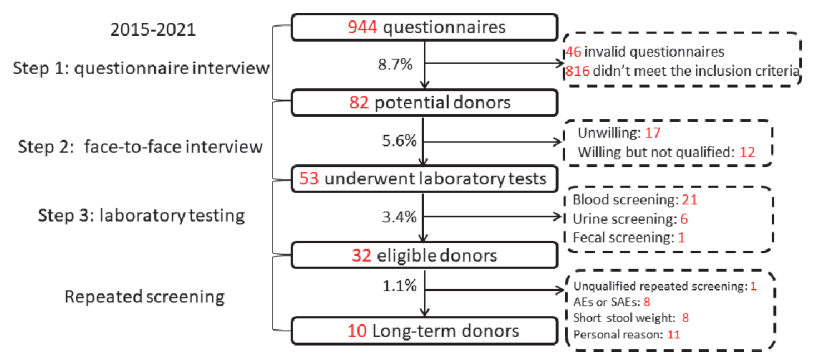

Abstract IDDF2021-ABS-0086 Figure 2

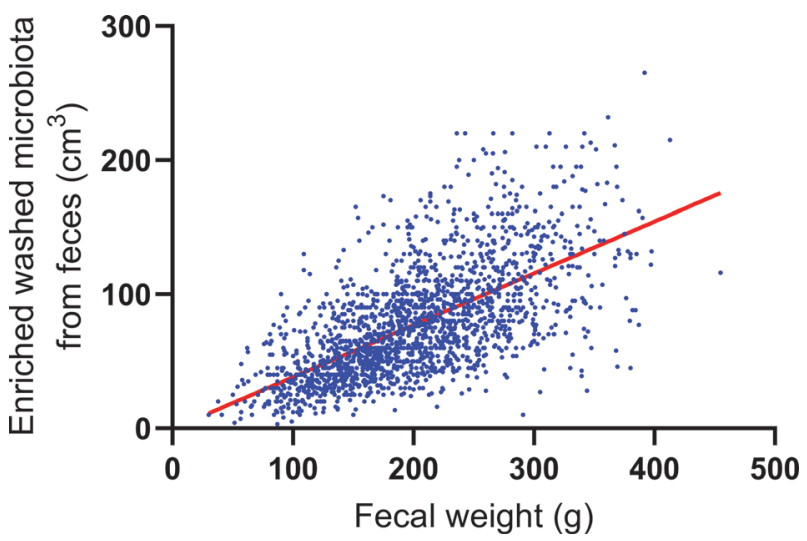

Abstract IDDF2021-ABS-0086 Figure 3

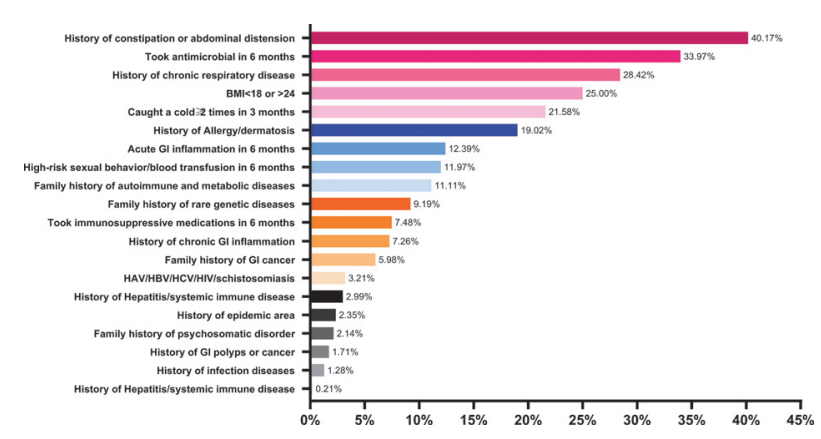

Abstract IDDF2021-ABS-0086 Figure 4

bank of the United States, Canada and South Korea. And we found that long-term donors have better microbiota output and compliance. The study found that it is feasible to screen qualified donors from a large sample by step-by-step screening. Through this programmed donor screening management process, convenient and repeatable donor screening can be realized.

\section{IDDF2021-ABS-0087 ENTEROCOCCUS AND BIFIDOBACTERIUM MAY BE RISK FACTORS FOR FECAL MICROBIOTA TRANSPLANTATION-RELATED ADVERSE EVENTS}

Weihong Wang*, Pan LI, Xia Wu, Faming Zhang. The Second Affiliated Hospital of Nanjing Medical University, China

\subsection{6/gutjnl-2021-IDDF.133}

Background Fecal microbiota transplantation (FMT) for inflammatory bowel disease (IBD) is an effective means of treatment, adverse events (AE) may influence the use of FMT and make patients worry about its safety. At present, there are still few studies on the safety of FMT in the world. Most of the previous studies on the safety of FMT focus on the gut microbiota from donors but ignore its from patients. This study is the first to analyze the risk factors of the occurrence and clinical efficacy of FMT-related AE from the perspective of patients.

Methods 102 IBD patients who had received FMT were included in the study. Clinical efficacy was followed up for 3 months and $\mathrm{AE}$ occurred during the course of disease was 
recorded. Fecal samples were collected before treatment for 16sRNA sequencing.

Results IBD patients were divided into AE group $(\mathrm{N}=34)$ and non-AE group $(\mathrm{N}=68)$ according to the occurrence of $\mathrm{AE}$. By sequencing the fecal microbiota of patients, it was found that enterococcus and bifidobacterium were the dominant microbiota genus in the AE group, which was higher than that in the non-AE group (IDDF2021-ABS-0087 Figure 1). Bifidobacterium and enterococcus are independent risk factors for the occurrence of FMT-related AE using univariate and multivariate logistic regression analysis(IDDF2021-ABS-0087 Figure 2,3). AE group is divided into fever subgroup and gastrointestinal complications (GC) subgroup, LEfSe analysis showed high abundance of enterococcus in fever group, high abundance of bifidobacterium in GC group (IDDF2021-ABS0087 Figure 4); Moreover, the abundance of bifidobacterium in the diarrhea subgroup in GC group was higher than that in the non-AE group (IDDF2021-ABS-0087 Figure 5). AE group was divided into non-response subgroup, response subgroup and remission subgroup according to clinical efficacy.
A
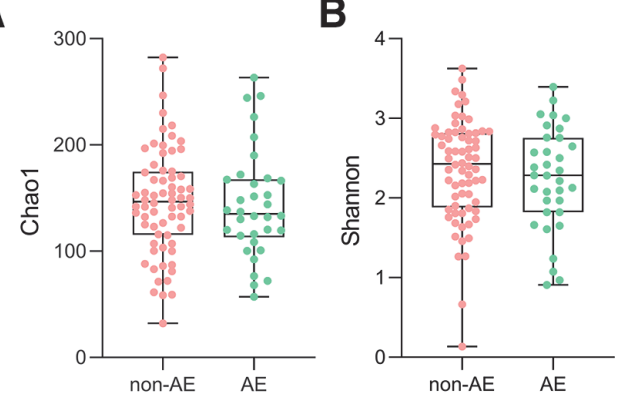

D

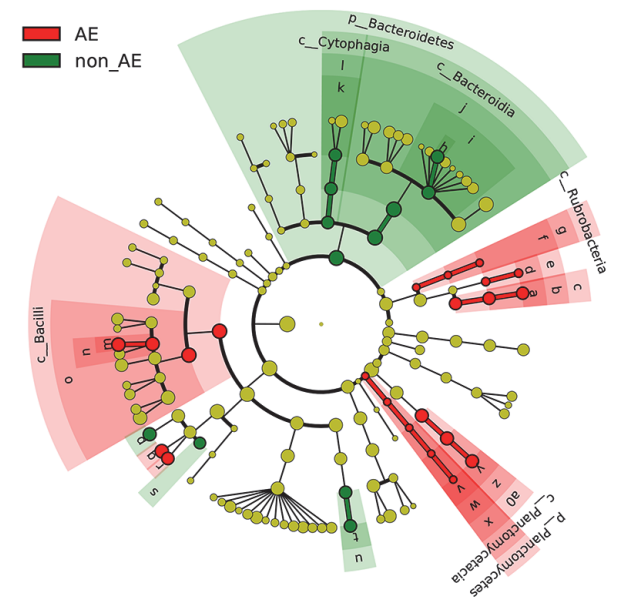

C

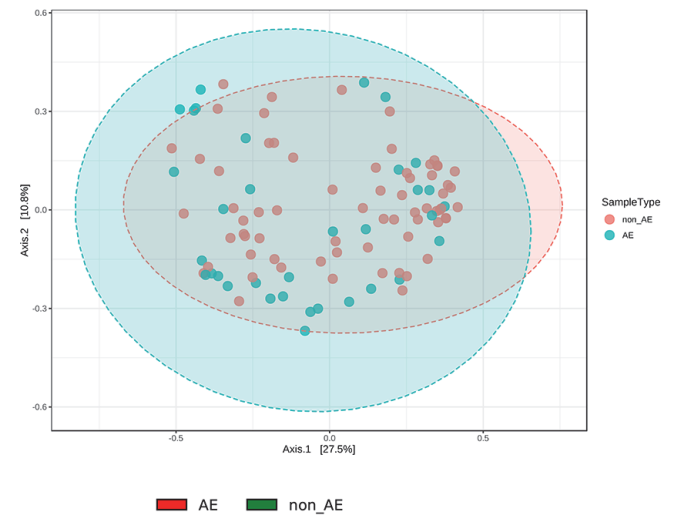

E
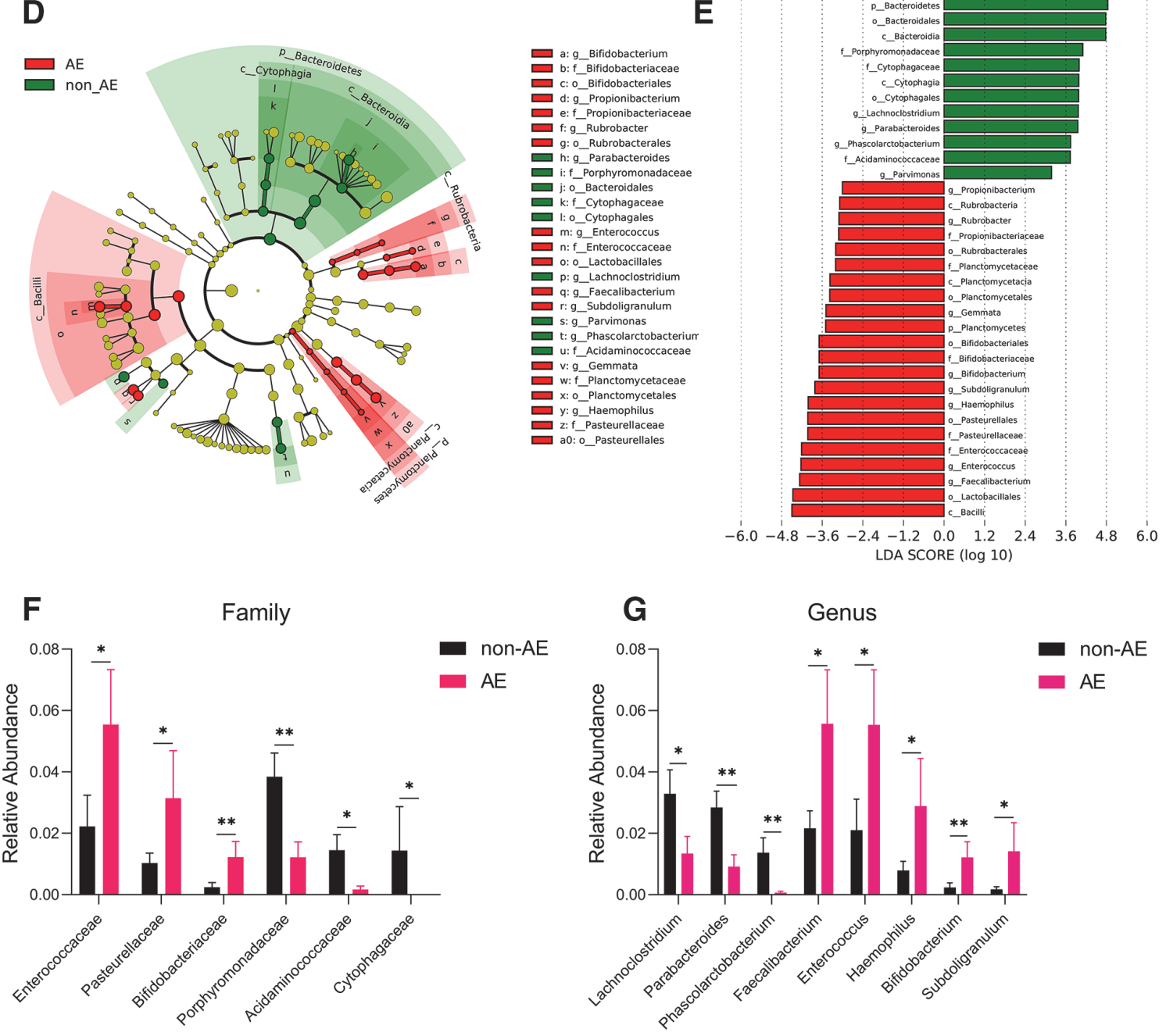

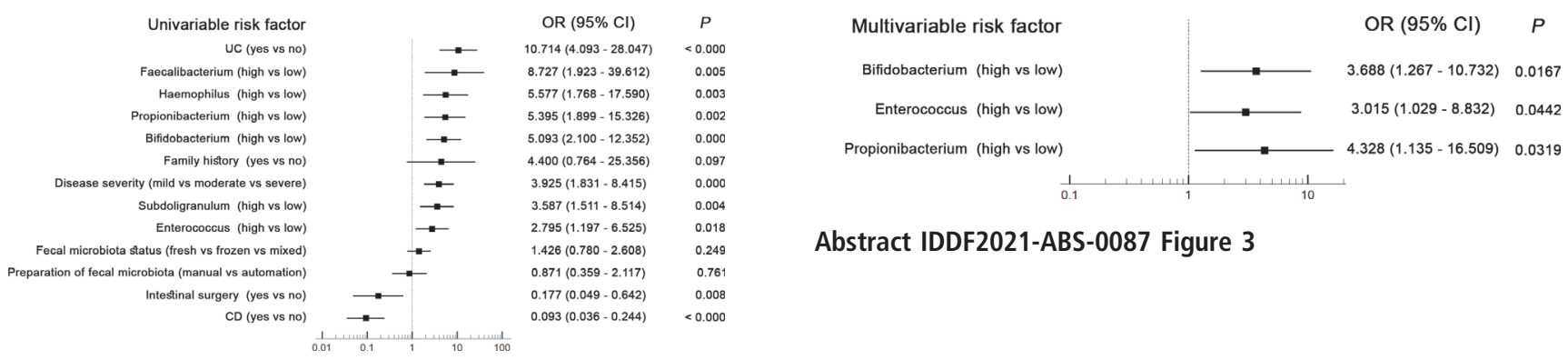

Abstract IDDF2021-ABS-0087 Figure 3

Abstract IDDF2021-ABS-0087 Figure 2

A

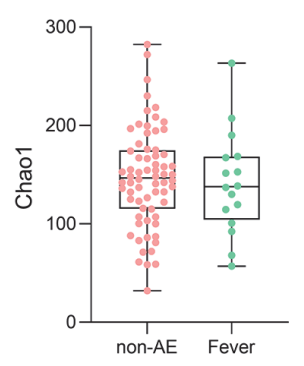

B

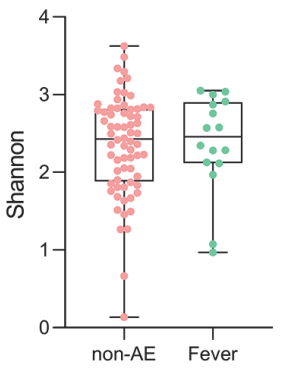

D

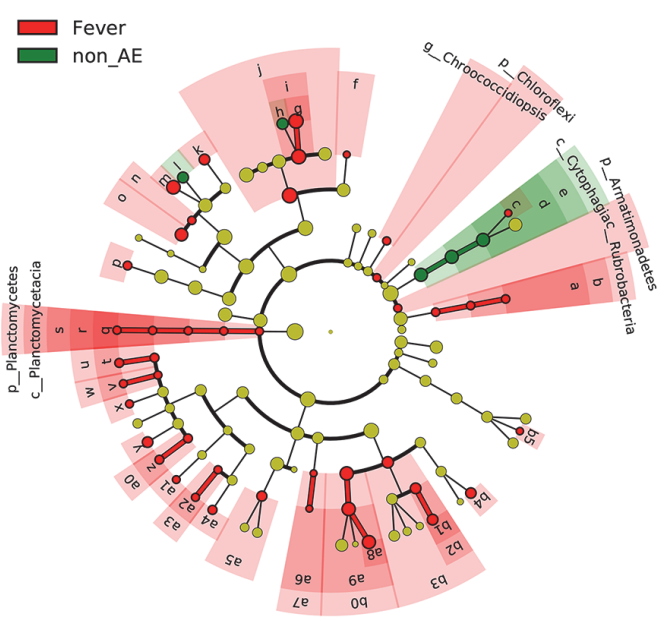
b: o_Rubrobacterales c: g_siphonobacter e: o_Cytophagales f: g_Exiguobacterium g: g_Enterococcus h: g_Vagococcus i: f_Enterococcaceae j: o_Lactobacillales k: g_Howardella

1. g_Anaerotruncus

m: g_Subdoligran

o: garvimonas

p: g_Candidatus_Stoquefichus

a: g_Gemmata

r: f_Planctomycetaceae

s: o_Planctomycetales

t: g_Bradyrhizobium

u: f_Bradyrhizobiaceae

v: g_Pseudochrobact

w: f_Brucellacea

y: g_Alihoeflea

z: g_Rhizobium

a0: f_Rhizobiaceae

a1: 9_Pannonib

a3: f Acetobact

a4: g_Elstera

a5: f Burkholderiaceae

a6: f_Desulfobulbacea

a7: o_Desulfobacteral

a8: 9_Haemophilus

a9:f_Pasteurellacea

b0: o_Pasteurellales
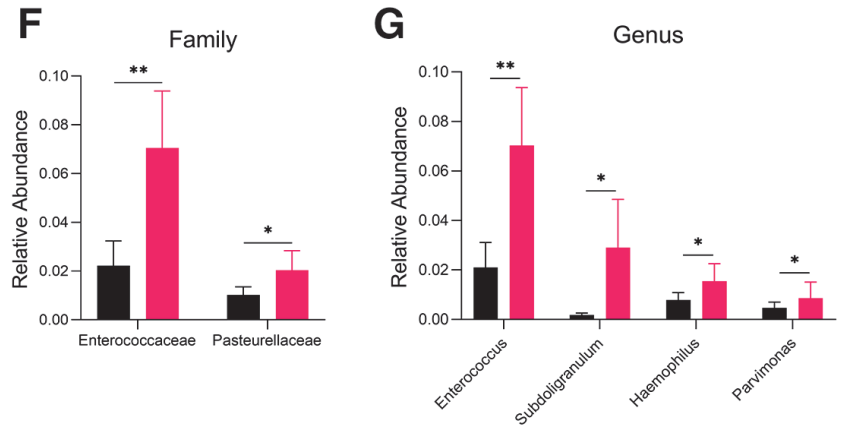

H

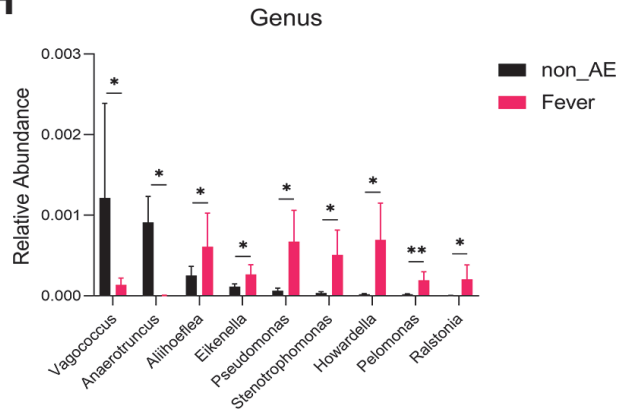

E

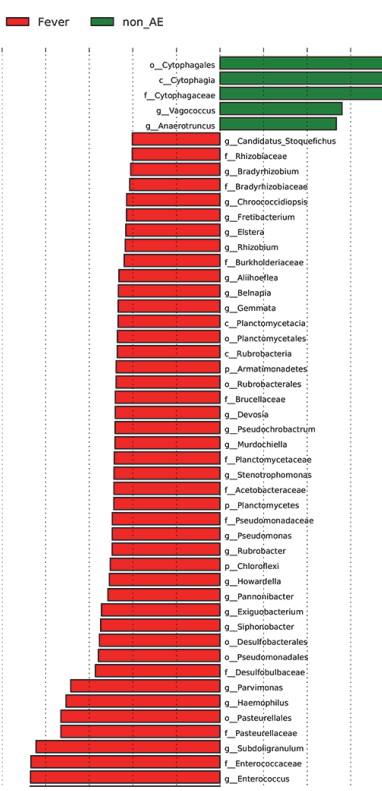

Genus

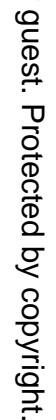


A

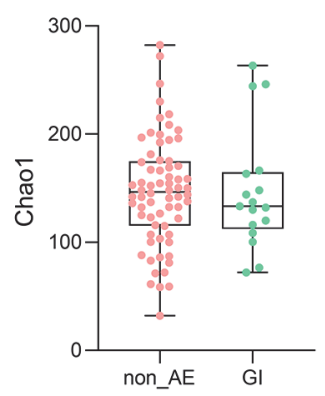

D

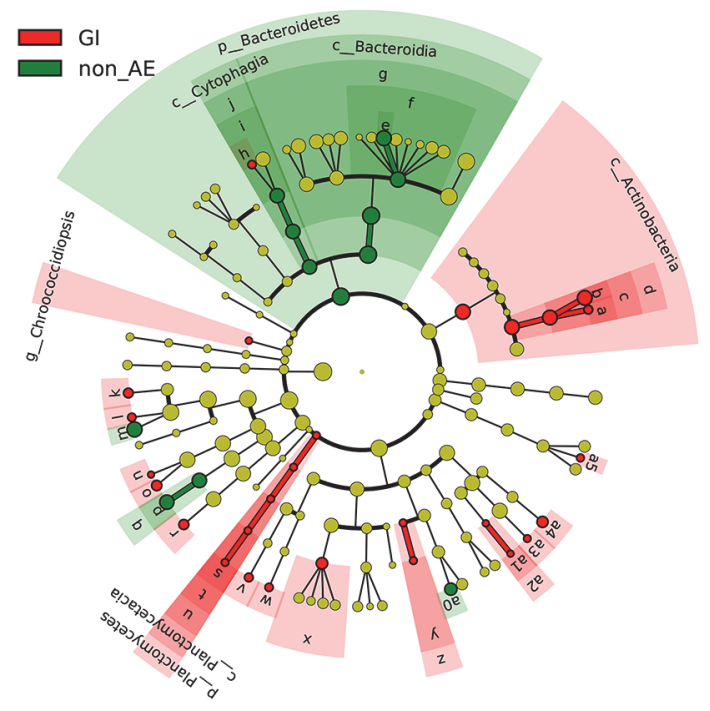

B
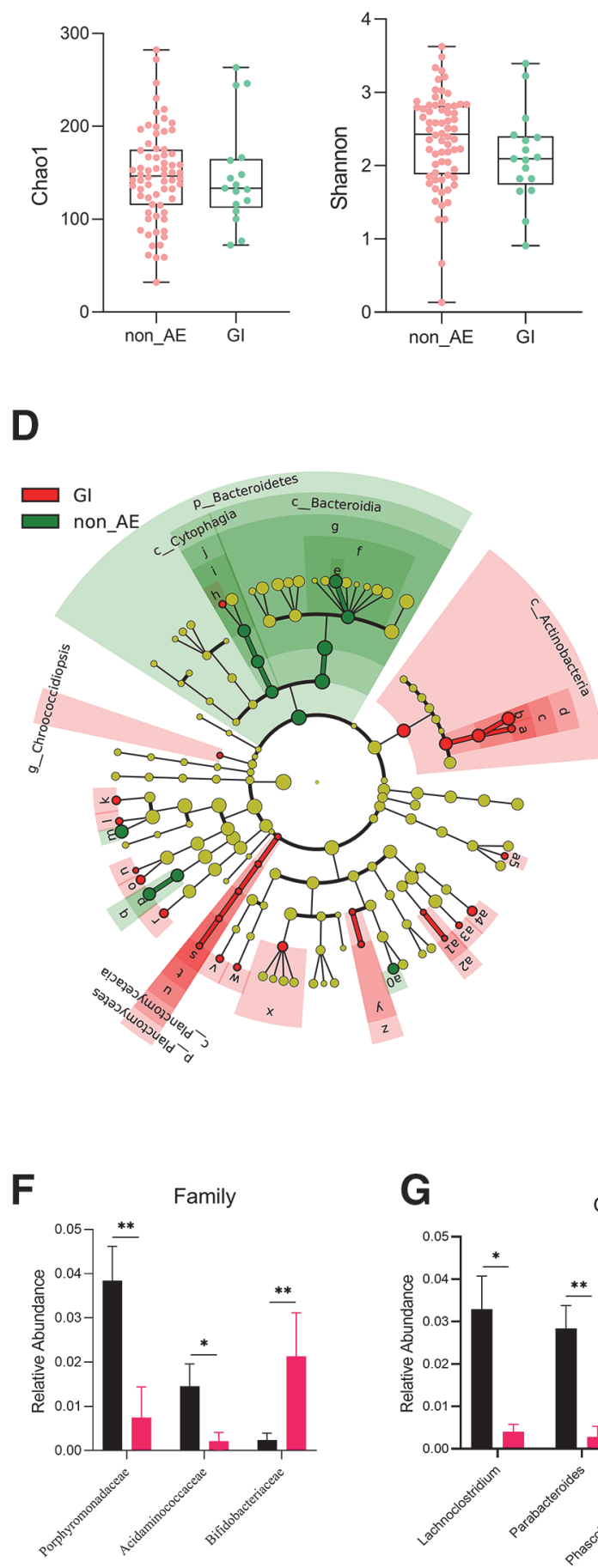

G

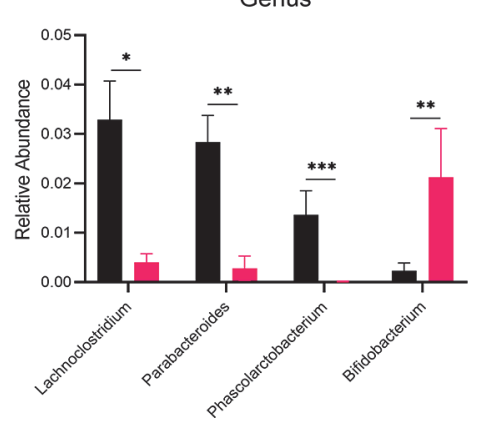

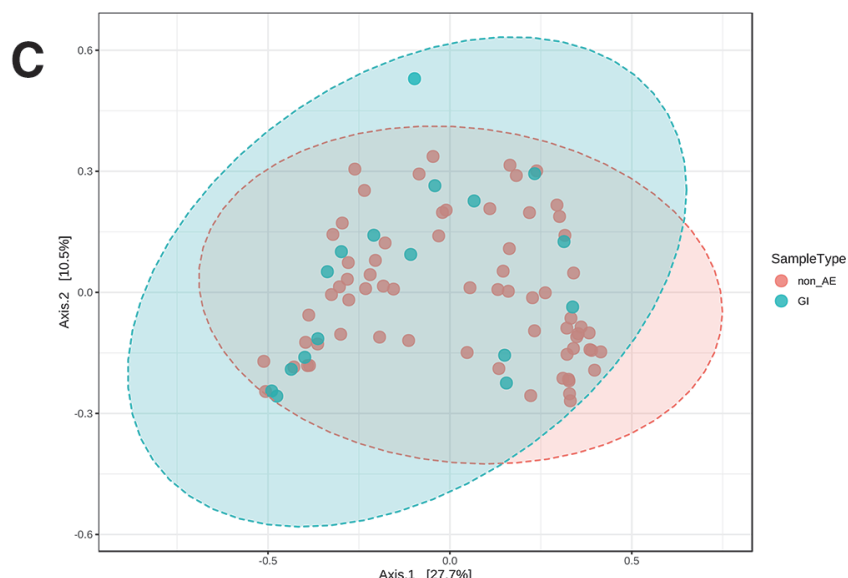

E

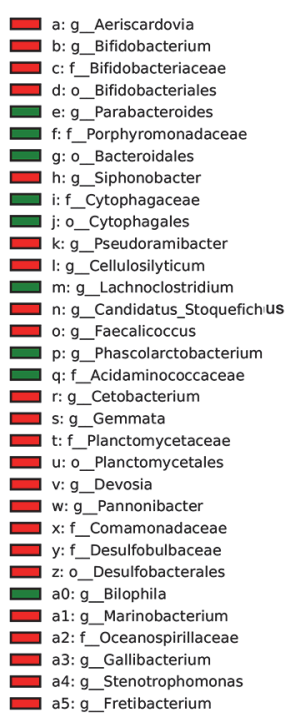

$\square$ GI $\square$ non_AE

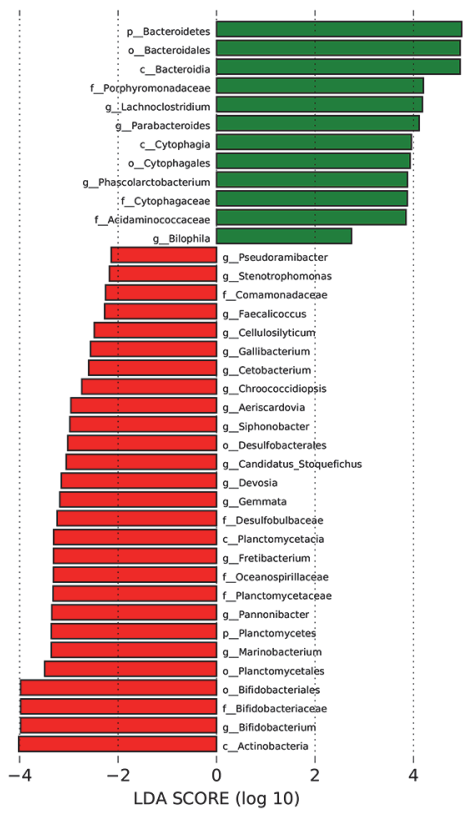

H

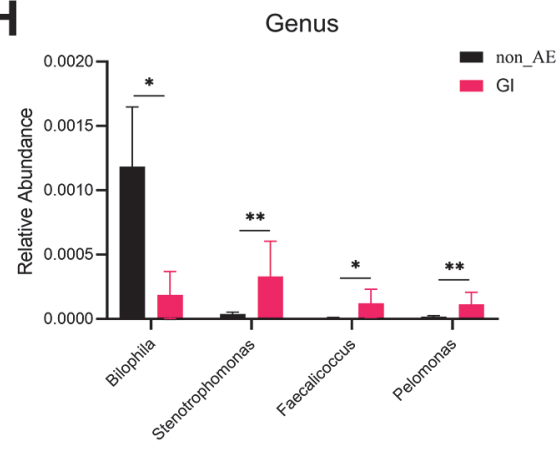

Abstract IDDF2021-ABS-0087 Figure 5 
A
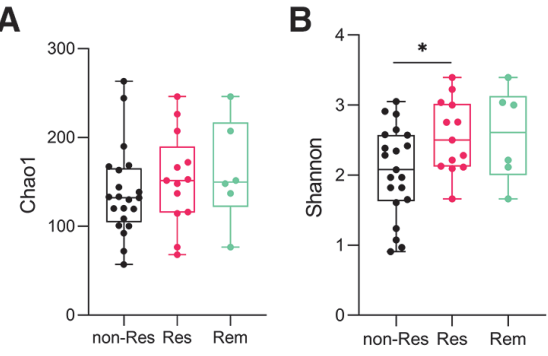

D

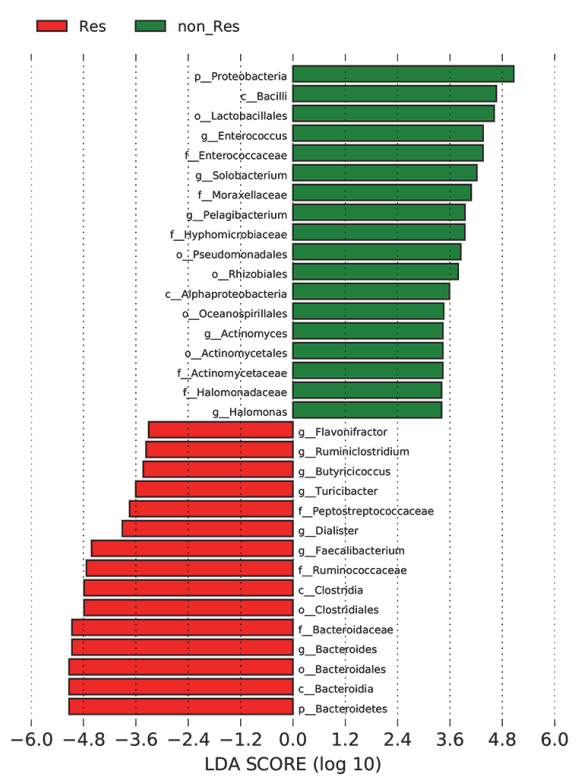

$\mathbf{F}$

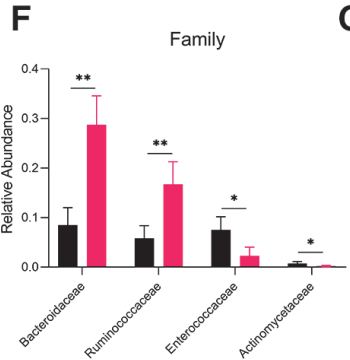

G

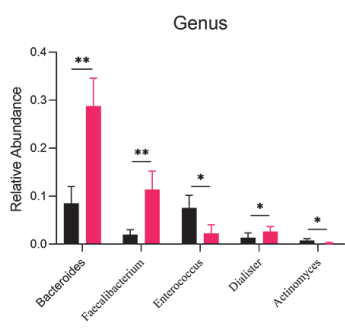

C

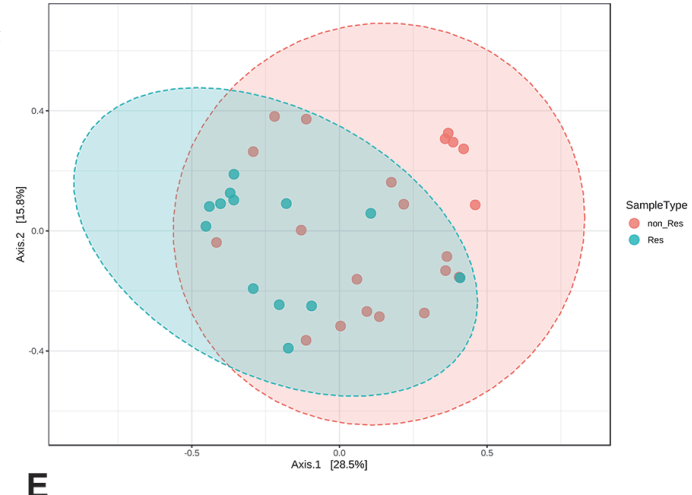

E

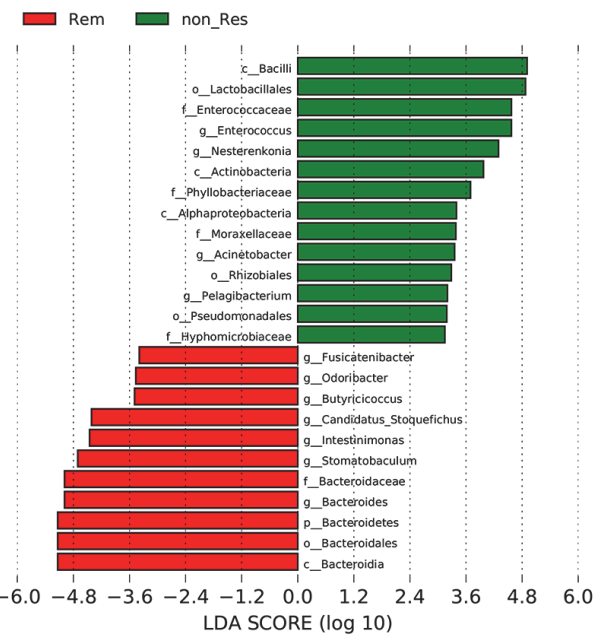

H
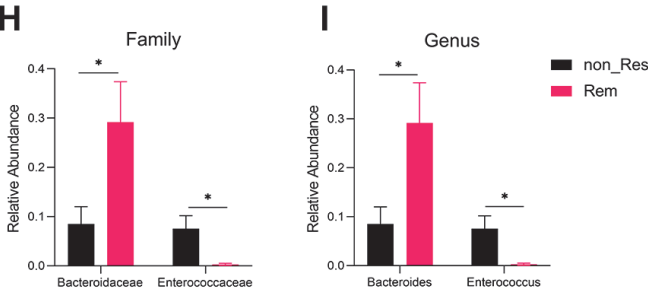

\section{Abstract IDDF2021-ABS-0087 Figure 6}

The abundance of enterococcus in the non-response group was higher than that in the other groups (IDDF2021-ABS0087 Figure 6).

Conclusions Enterococcus and bifidobacterium may be risk factors for FMT-related AE, and the high abundance of enterococcus in patients may affect the efficacy of FMT.

\section{IDDF2021-ABS-0090 RESEARCH ON APPLICATION OF WEXNER SCORE AND MAGNETIC RESONANCE DEFECOGRAPHY IN PATIENTS WITH FUNCTIONAL CONSTIPATION}

Anh Tran Ngoc*, Chi Duong Thi Mai. Hanoi Medical University Hospital, Vietnam

\subsection{6/gutjnl-2021-IDDF.134}

Background To describe some clinical characteristics, Wexner score, and magnetic resonance defecography in patients with functional constipation.
Methods Describing cross-study. Forty-two (42) patients were diagnosed with functional constipation according to ROME IV criteria. Magnetic resonance defecography and evaluation of the severity of constipation according to Wexner score were indicated in these patients from $01 / 2020$ to $06 / 2021$ at Hanoi Medical University Hospital.

Results The mean duration of constipation was $6.1 \pm 5.9$ years. Dyschezia and prolonged defecation were common symptoms (IDDF2021-ABS-0090 Table 1). Decreased frequency of bowel movements only accounted for $57.1 \%$ (IDDF2021-ABS-0090 Table 2). Magnetic resonance defecography detected $24 / 42$ patients with rectocele; $16 / 42$ patients with anismus; $15 / 42$ patients with rectal prolapse, and 20/42 patients with uterus, bladder prolapse (IDDF2021-ABS-0090 Table 4). Incomplete defecation and assistance were common in rectocele. The group with rectocele and rectal prolapse experienced a longer duration of constipation. Decreased frequency of bowel movements was common in the group with anismus. The mean Wexner score was 13.3 44.0 (IDDF2021- 